\section{PLEOCYTOSIS PREDICTS BORRELIA ETIOLOGY IN CHILDREN WITH FACIAL PALSY}

Heidi Pöyhönen, Ville Peltola, Jukka Hytönen* and Tuire Lähdesmäki.

Departments of Paediatrics and Adolescent Medicine and *Clinical microbiology, Turku University Hospital and University of Turku, Turku, Finland.

Background: Lyme borreliosis (LB) is a common cause of acute facial palsy in children living in Borrelia burgdorferi endemic areas. LB can be detected by specific antibodies in serum and cerebrospinal fluid (CSF). Need for lumbar puncture in diagnostics of LB in children with facial palsy has been questioned.

Aim: We examined the utility of diagnostic lumbar puncture and sensitivity and specificity of CSF pleocytosis in LB diagnostics among children with acute facial palsy.

Methods: We collected medical records and laboratory data of patients under 17 years of age $(n=94)$ treated for facial palsy in the Department of Paediatrics and Adolescent Medicine, Turku University Hospital, in years 2002-2016. Positive IgM or IgG antibodies against flagellar antigen, positive C6 index, positive $B$. burgdorferi PCR in CSF, or positive intrathecal antibody index were considered as signs of definite LB diagnosis.

Table 1. Patient characteristics (n=94). CSF cerebrospinal fluid.

\begin{tabular}{|l|r|r|}
\hline Characteristic & & Number (\%) \\
\hline \multirow{3}{*}{ Sex } & Male & $46(49 \%)$ \\
\cline { 2 - 3 } & Female & $48(51 \%)$ \\
\cline { 2 - 3 } & Right & $48(51 \%)$ \\
\cline { 2 - 3 } $\begin{array}{l}\text { Number of facial palsy } \\
\text { episodes }\end{array}$ & Left & $43(46 \%)$ \\
\cline { 2 - 3 } & 1 & $3(3 \%)$ \\
\cline { 2 - 3 } & 2 & $89(95 \%)$ \\
\hline CSF sample taken & 3 & $4(4 \%)$ \\
\hline
\end{tabular}

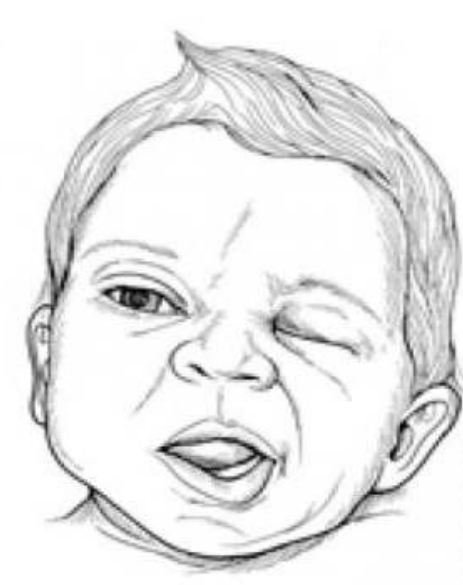

Results: Lumbar puncture was performed to 84/94 patients. Forty-two patients $(50 \%)$ had CSF pleocytosis $(>5 \times 10 \mathrm{E} 6$ cells/L). Diagnostic criteria of LB were fulfilled by 30 facial palsy patients with pleocytosis and by 4 without pleocytosis. The sensitivity and specificity of pleocytosis to predict LB was $88 \%$ and $60 \%$, respectively. Positive and negative predictive values were $71 \%$ and $90 \%$. LB was detected only by serology in 13 $(38 \%)$, only by CSF testing in $10(29 \%)$, and by both serum and CSF analysis in $11(32 \%)$ of 34 LB patients.

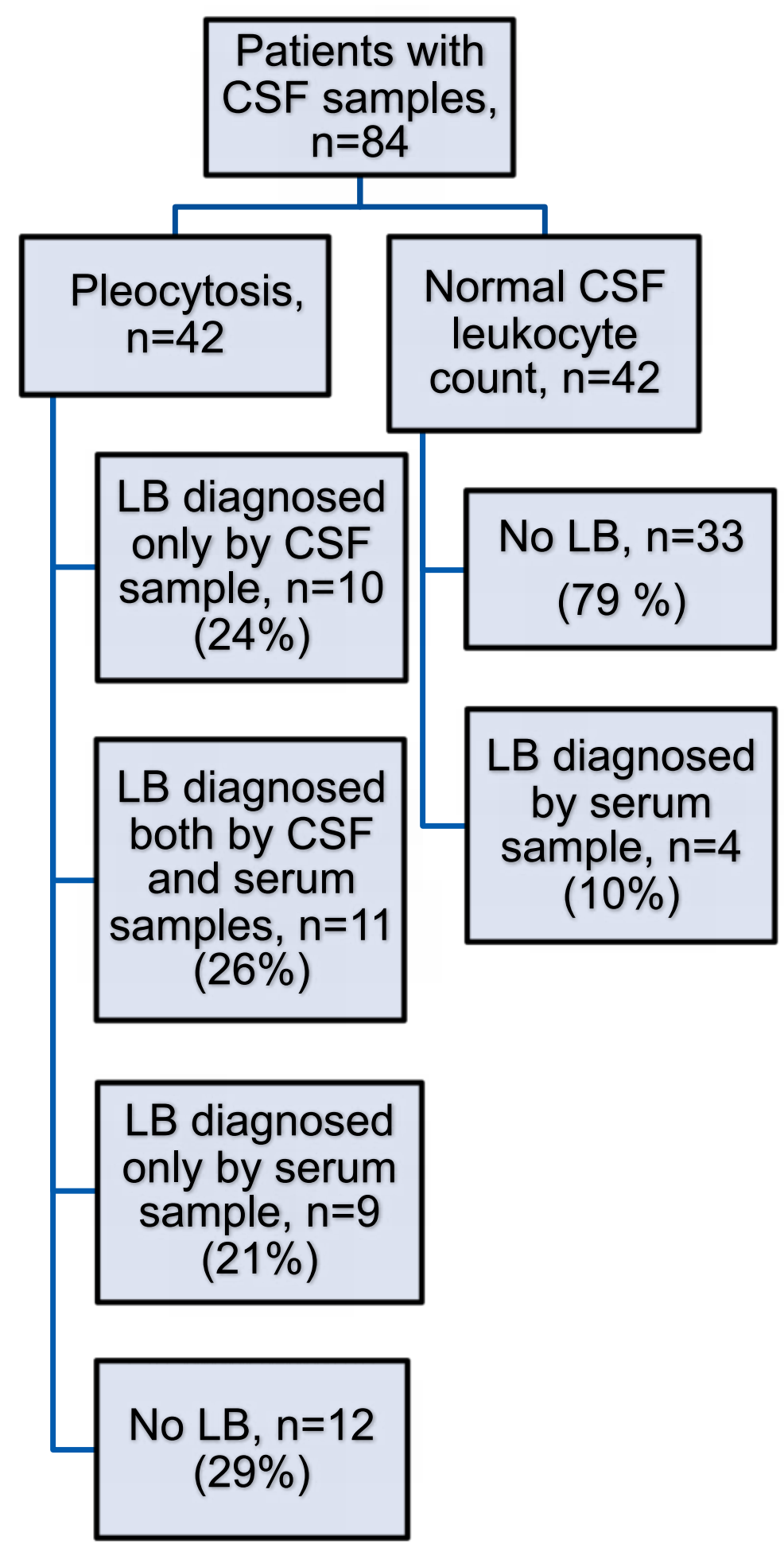

Figure 1. Lyme borreliosis (LB) detected by serology and/or findings in cerebrospinal fluid (CSF) in children with facial palsy with or without CSF pleocytosis.
Conclusions: Lumbar puncture is necessary in diagnostics of LB in children with facial palsy, because serology can be negative in the early phase of illness. CSF pleocytosis predicts LB. In endemic areas antimicrobial treatment against LB can be started based on pleocytosis even before antibody results are available.

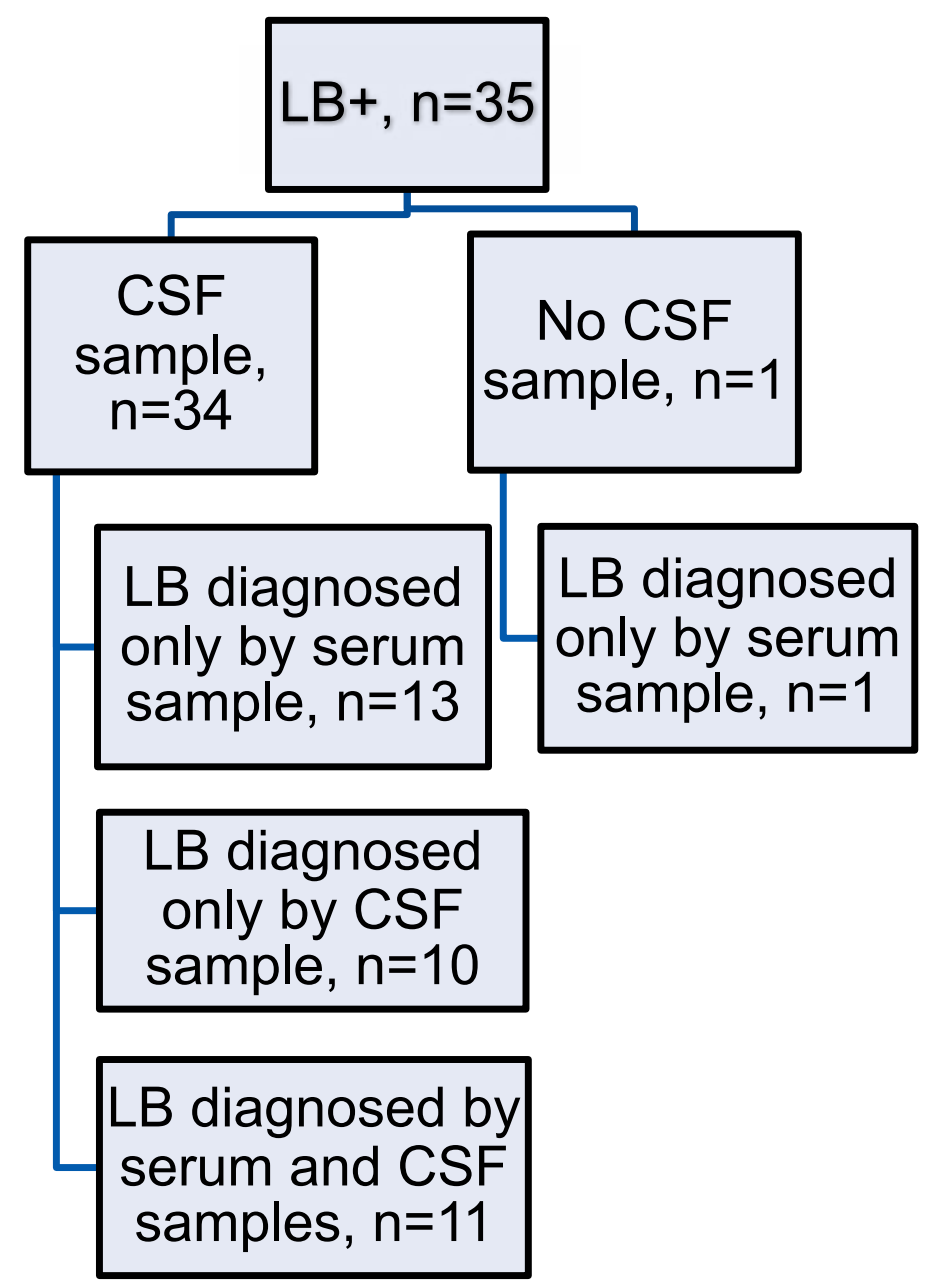

Figure 2. Definite diagnosis of Lyme borreliosis (LB) was based on serology and/or findings in cerebrospinal fluid (CSF).

\begin{tabular}{ll}
\hline \multicolumn{2}{c}{ Pleocytosis } \\
\hline Sensitivity & $88 \%$ \\
Specificity & $60 \%$ \\
PPV & $71 \%$ \\
NPV & $90 \%$ \\
\hline
\end{tabular}

Table 2. Sensitivity, specificity, positive predictive value (PPV) and negative predictive values (NPV) of pleocytosis for Lyme borreliosis in children with facial palsy. 